\title{
GZ Cancri: A cataclysmic variable at the lower edge of the period gap ${ }^{\star}$
}

\author{
C. Tappert ${ }^{1}$ and A. Bianchini ${ }^{2}$ \\ 1 Departamento de Física, Grupo de Astronomía, Universidad de Concepción, Casilla 160-C, Concepción, Chile \\ 2 Dipartimento di Astronomia, Università di Padova, Vicolo dell’Osservatorio 2, 35122 Padova, Italy
}

Received 11 November 2002 / Accepted 6 February 2003

\begin{abstract}
We present calibrated photometry and time-resolved spectroscopy of the cataclysmic variable GZ Cnc. Radial velocities of the $\mathrm{H} \alpha$ emission line reveal an orbital period of $0.08825(28) \mathrm{d}$, or 2.118(07) h, placing the system at the lower edge of the period gap. One of our observations catches the system on the rise to an overlooked, possibly small-amplitude, outburst. At certain phases during this stage the profiles of the emission lines are distorted by a high velocity absorption component. We discuss this phenomenon in the context of the recent suggestion that the long-term lightcurve of GZ Cnc resembles those of intermediate polars.
\end{abstract}

Key words. stars: individual: GZ Cnc - stars: novae - cataclysmic variables - stars: fundamental parameters - binaries: general

\section{Introduction}

Cataclysmic variables (CVs) are close interacting binaries with a white dwarf as primary component and usually a late-type main-sequence star as secondary. In the course of their evolution from wide detached binaries, angular momentum loss thought to be driven by magnetic braking and gravitational radiation has shrunk the Roche lobe of the secondary into contact with the stellar surface. This causes mass transfer via the Lagrange point $L_{1}$ into the gravitational well of the primary. In the absence of strong magnetic fields, an accretion disc around the white dwarf is formed, which is also the major source of the typical emission line spectrum. For a comprehensive overview on CVs see Warner (1995).

GZ Cnc (= RX J0915.8+0900 = Tmz V034) was discovered independently as a variable star and a ROSAT source (Takamizawa 1998; Bade et al. 1998). A spectrum was presented by Jiang et al. (2000), showing a blue continuum and moderately strong emission lines. Although the spectral coverage went up to $8200 \AA$, no spectroscopic signatures of the secondary star were detected. Kato et al. (2001) obtained lightcurves of the star during decline in outburst. Their longest time series spans $\sim 3.1 \mathrm{~h}$ and does not show any apparent periodic variations. Both the spectroscopic and the photometric properties, together with the outburst behaviour (slow rise, small amplitude; Kato et al. 2001) therefore point to an SS Cygtype dwarf nova with an orbital period $>3 \mathrm{~h}$. In a recent paper

Send offprint requests to: $\mathrm{C}$. Tappert,

e-mail: claus@gemini.cfm.udec.cl

* Based on observations made at the European Southern Observatory.
Kato et al. (2002) investigate the long-term lightcurve in more detail, and emphasise similarities to those of intermediate polars. Again, this would favour a long orbital period, as the vast majority of those systems is found above the period gap (Ritter \& Kolb 1998).

We here present time-resolved spectroscopy in order to derive the orbital period, which, in fact, turns out to be less than 3 h. In Sect. 2 we describe the observations and the reduction process. The results are presented in Sect. 3 and subsequently discussed in Sect. 4. We complete our paper with a short conclusion in Sect. 5.

\section{Observations and reductions}

The data were taken on January 16-18, 2001, and on March 26-28, 2002, with the DFOSC system at the $1.54 \mathrm{~m}$ Danish telescope at ESO, La Silla, Chile. The spectroscopy was performed using grism \#7, yielding a wavelength range of 3800-7000 A. A slit width of 1'.5 gave a spectral resolution of $4.9 \AA$. The measurements were accompanied by He-Ne arc spectra for wavelength calibration and by spectrophotometric standards for flux calibration. Between each row of spectroscopic measurements, photometric exposures were taken in the $V$ passband. Additionally, calibrated $B V R$ photometry was obtained on 2002-03-26. Table 1 presents the observation log.

The reduction process included bias and flatfield correction, as well as wavelength and flux calibration, which were performed with IRAF routines (Tody 1993). Further analysis of the spectroscopic data was done with ESO-MIDAS (Warmels 1992), while the photometry made use of the IRAF daophot package and the standalone daomatch and daomaster routines 
Table 1. Overview of the observations. $n_{\text {data }}$ gives the number of data points, $t_{\exp }$ the individual exposure time, and $\Delta t$ the time range covered by the observations.

\begin{tabular}{|c|c|c|c|c|}
\hline date & $n_{\text {data }}$ & $t_{\exp }[\mathrm{s}]$ & $\Delta t[\mathrm{~h}]$ & Notes \\
\hline \multicolumn{5}{|c|}{ Spectroscopic data: } \\
\hline 2001-01-16 & 12 & 600 & 2.40 & \\
\hline 2001-01-17 & 13 & 600 & 2.48 & \\
\hline 2001-01-18 & 11 & 600 & 2.29 & \\
\hline $2002-03-27$ & 16 & 300 & 1.93 & \\
\hline 2002-03-28 & 26 & 300 & 3.01 & \\
\hline \multicolumn{5}{|c|}{ Photometric data: } \\
\hline 2001-01-16 & 4 & 20 & 2.26 & \\
\hline 2001-01-17 & 3 & 20 & 2.05 & \\
\hline 2001-01-18 & 4 & 20 & 1.62 & \\
\hline 2002-03-26 & 1 & $60,20,15$ & - & $B, V, R$ \\
\hline 2002-03-27 & 7 & 20 & 1.78 & \\
\hline 2002-03-28 & 8 & 20 & 2.36 & \\
\hline
\end{tabular}

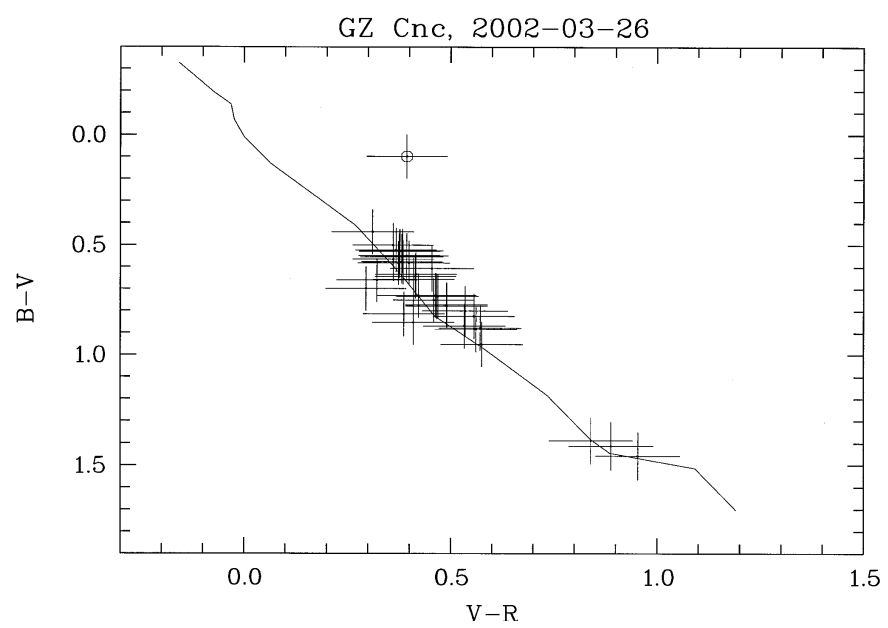

Fig. 1. Colour-colour diagram of the field of GZ Cnc, consisting of 38 field stars and GZ Cnc itself (marked by a circle). The range of magnitudes is $B=14.4-18.5, V=13.7-17.7, R=13.4-17.2$. The solid line marks the Bessell (1990) main sequence.

(Stetson 1992). The photometric calibration was obtained by comparison with Landolt (1992).

\section{Results}

\subsection{Photometry}

Only on 2002-03-26 direct calibrated photometric data were obtained in $B, V$, and $R$ passbands. The resulting colours are

$B-V=0.097(99), V-R=0.394(97)$,

at $V=15.471(85)$,

which lie in the usual range occupied by dwarf novae (Echevarría 1984), and put it clearly away from the main sequence (Fig. 1).

For the other nights, calibrated $V$ magnitudes for $\mathrm{GZ} \mathrm{Cnc}$ were computed with respect to an average lightcurve composed of 5 stars which could be measured in all frames and proved to be constant within the errors. The resulting magnitudes are given in Table 2. During the 2002 observing run the system
Table 2. Calibrated magnitudes for GZ Cnc. Note that the date marks the start of the night in local time. The errors in Cols. 3 and 4 result from the combination of the photometric error and the error of the differential lightcurve, with the exception of the value from 2002-03-26 which represents the error of the photometric calibration only.

\begin{tabular}{llll}
\hline \hline date & HJD & $V_{\min }$ & $V_{\max }$ \\
\hline $2001-01-16$ & 2451926 & $15.460(38)$ & $15.345(38)$ \\
$2001-01-17$ & 2451927 & $15.393(38)$ & $15.228(38)$ \\
$2001-01-18$ & 2451928 & $15.144(38)$ & $14.812(38)$ \\
$2002-03-26$ & 2452360 & $15.471(85)$ & \\
$2002-03-27$ & 2452361 & $15.648(28)$ & $15.439(27)$ \\
$2002-03-28$ & 2452362 & $15.698(27)$ & $15.357(27)$ \\
\hline
\end{tabular}

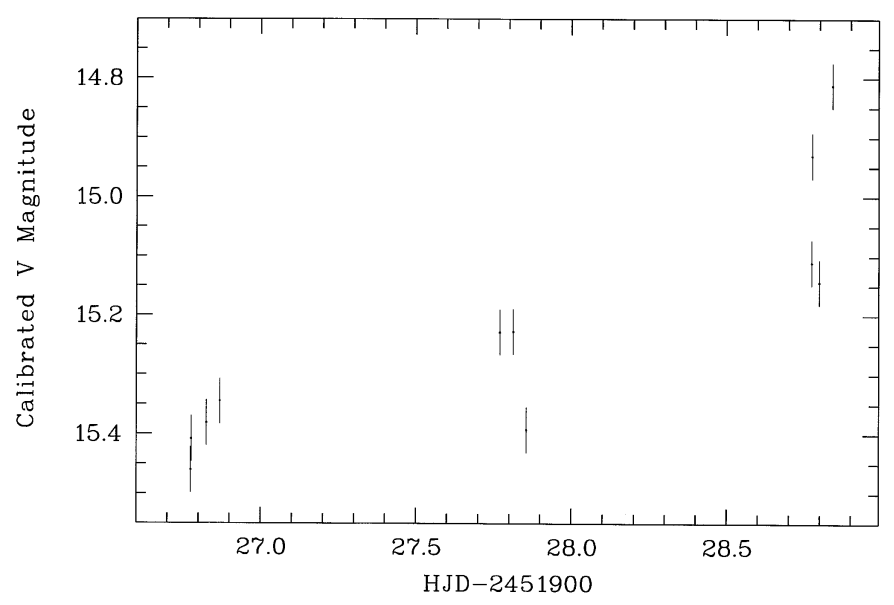

Fig. 2. Photometric data of GZ Cnc for the 2001 run.

appears to have been in a stable quiescent state, with variations $\Delta m_{V} \sim 0.3 \mathrm{mag}$ probably due to flickering or an additional continuum source (e.g., the bright spot). A comparison with Kato et al. (2002) shows that our observations lie close to two recorded outbursts on 2002-03-11 and 2002-04-02. The 2001 data, however, clearly show a brightness increase, which could be interpreted as the onset of an outburst (see Fig. 2), although to our knowledge none has been registered in the subsequent days. However, the coverage of GZ Cnc contains a lot of gaps. For the year 2001, only 11 observations are recorded in the VSNET database ${ }^{1}$, among them one single positive detection. A weak outburst could thus easily have been missed.

The increase rate of roughly $0.2 \mathrm{mag} \mathrm{d}^{-1}$ in our data fits well with the outburst recorded by Kato et al. (2001), that showed a rate of $\sim 0.25 \mathrm{mag} \mathrm{d}^{-1}$ over 5 nights. This lends further support to the idea of an overlooked outburst in 2001.

\subsection{Properties of the average spectra}

The spectrum of GZ Cnc (Fig. 3) resembles that of a dwarf nova, with dominating emission lines from the Balmer and He I series. Further lines include He II 24686 , as well as Fe II $\lambda 5172$. Table 3 summarises the identified lines and their equivalent widths. The emission lines are of rather moderate strength (e.g., Williams 1983), which indicates a moderately

\footnotetext{
1 The archive of the VSNET mailing list is located at http://www. kusastro.kyoto-u.ac.jp/vsnet/Mail
} 


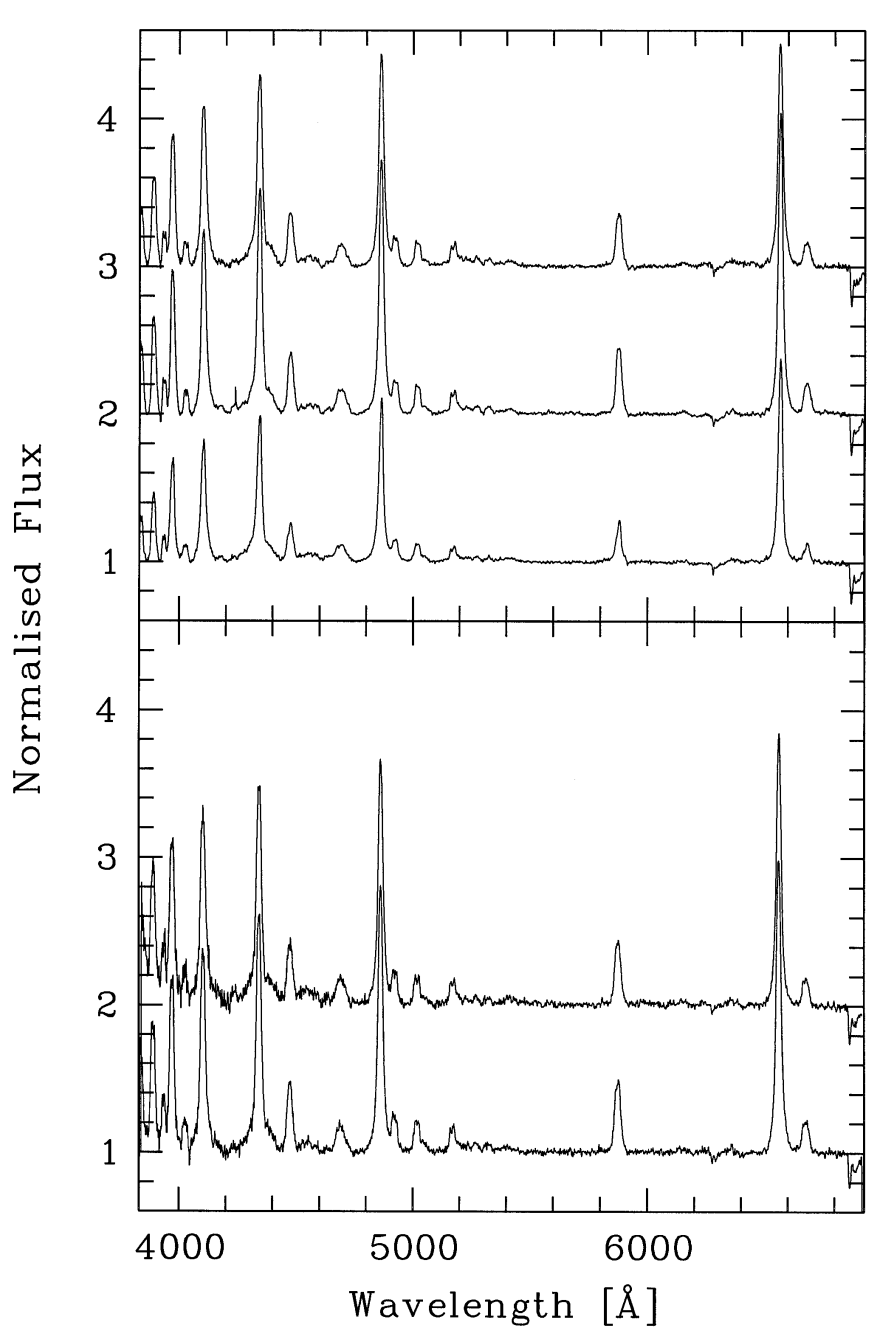

Fig. 3. Average normalised spectra of GZ Cnc. Above: spectra from 2001-01-16, 2001-01-17, and 2001-01-18 (from top to bottom). The former two have been displaced by 2 and 1 units in vertical direction. The small spike at $\lambda=4240 \AA$ in the 2001-01-17 spectrum is an artifact, the absorption lines in the red part of the spectrum are atmospheric. Below: spectra from 2002-03-27 (top, displaced vertically by 1 unit) and 2002-03-28 (bottom).

high mass-transfer rate (Fig. 6 in Patterson 1984). As noted by Kato et al. (2002), He II appears to be somewhat stronger than in most dwarf novae, and especially those with a short orbital period.

The slow rise in the photometry during the 2001 run is also roughly reflected by the equivalent widths of the emission line, which first show a slight rise from the first to the second night, followed by a steep decline on the third night (Table 3, Fig. 3). It appears thus that this variation is indeed due to a brightening of the disc continuum.

\subsection{Period analysis}

The Doppler variation of the emission lines caused by the orbital motion of the system has been measured with a Gaussian fit to the whole $\mathrm{H} \alpha$ line profile. Radial velocities were computed with respect to the O I night sky emission line
Table 3. Identified emission lines and their equivalent widths. Only lines which were detected in all five averaged spectra are shown. Column 1 gives the average wavelength position of the lines in the spectra, Col. 2 the identification, and Col. 3 the corresponding rest wavelength. Columns $4-8$ contain the measured equivalent widths for the five nights. In two cases the values for the blend of two lines are given for the stronger line: Ca II $\lambda 3934+\mathrm{H} \epsilon$ (note that $\mathrm{H} \epsilon$ itself already includes the other Ca II line) and $\mathrm{H} \beta+\mathrm{He} \mathrm{I} \lambda 4922$.

\begin{tabular}{|c|c|c|c|c|c|c|c|}
\hline \multirow[t]{3}{*}{$\lambda[\AA]$} & \multirow[t]{3}{*}{ ID } & \multirow[t]{3}{*}{$\lambda_{0}[\AA]$} & \multicolumn{5}{|c|}{$W_{\lambda}[\AA]$} \\
\hline & & & \multicolumn{3}{|c|}{ 2001-01 } & \multicolumn{2}{|c|}{$2002-03$} \\
\hline & & & 16 & 17 & 18 & 27 & 28 \\
\hline 3890.5 & $\mathrm{H} \zeta$ & 3889.1 & -15 & -17 & -10 & -15 & -16 \\
\hline 3935.9 & $\mathrm{Ca}$ II & 3933.7 & & & & & \\
\hline 3971.0 & $\mathrm{H} \epsilon$ & 3970.1 & -26 & -30 & -18 & -28 & -28 \\
\hline 4026.0 & $\mathrm{He} \mathrm{I}$ & 4026.2 & -3 & -4 & -2 & -3 & -5 \\
\hline 4103.4 & $\mathrm{H} \delta$ & 4101.7 & -33 & -36 & -24 & -35 & -44 \\
\hline 4341.7 & $\mathrm{H} \gamma$ & 4340.5 & -45 & -46 & -38 & -31 & -53 \\
\hline 4474.1 & $\mathrm{He} \mathrm{I}$ & 4471.5 & -11 & -12 & -7 & -9 & -13 \\
\hline 4688.9 & He II & 4685.7 & -10 & -8 & -7 & -9 & -8 \\
\hline 4862.4 & $\mathrm{H} \beta$ & 4861.3 & -53 & -60 & -39 & -59 & -64 \\
\hline 4920.4 & $\mathrm{He} \mathrm{I}$ & 4921.9 & & & & & \\
\hline 5018.1 & $\mathrm{He} \mathrm{I}$ & 5015.7 & -6 & -7 & -5 & -8 & -8 \\
\hline 5172.2 & $\mathrm{Fe}$ II & 5169.0 & -4 & -4 & -3 & -4 & -5 \\
\hline 5268.7 & Fe II & 5272.4 & -1 & -1 & -1 & -1 & -1 \\
\hline 5321.4 & $\mathrm{Fe}$ II & 5325.6 & -1 & -1 & -1 & -2 & -1 \\
\hline 5876.7 & $\mathrm{He} \mathrm{I}$ & 5875.6 & -12 & -14 & -8 & -15 & -16 \\
\hline 6563.9 & $\mathrm{H} \alpha$ & 6562.8 & -46 & -59 & -38 & -53 & -58 \\
\hline 6677.8 & $\mathrm{He} \mathrm{I}$ & 6678.1 & -5 & -7 & -3 & -6 & -6 \\
\hline
\end{tabular}

at $\lambda 5577 \AA$. As the mean velocity showed strong nightly variations, and the respective time ranges proved to cover more than one orbit, we normalised the velocity data individually by subtracting the respective mean velocities.

The search for periodicities was conducted using the Scargle (1982) and AOV (Schwarzenberg-Czerny 1989) algorithms as implemented in MIDAS' tsa context. Unfortunately, the time span between both observing runs proved to be too long for an analysis of the combined data, so that the runs had to be investigated individually. The Scargle algorithm produced five significant peaks in the range $f=9.35-13.35 \mathrm{cyc} \mathrm{d}^{-1}$. The frequencies corresponding to the highest peaks were 10.34 for the 2001 data and 11.34 for 2002 . The AOV method in both cases picked out $f \approx 11.35$. For the 2002 data, this peak is well separated from the next highest one, for 2001 the two peaks of 10.34 and 11.34 are very close (Fig. 4, lower and upper plot).

In most CVs, additional emission components distort the symmetric, disc-borne, line profile (Tappert \& Hanuschik 2001), with the type and strength of this distortion being likely to depend on the luminosity state of the system (e.g., Tappert 1999). Since on 2001-01-18 both the photometric magnitudes (Table 2) and the emission line equivalent widths (Table 3 ) differ strongly from those of the other nights in this run, we also conducted a period analysis for the data of the first two nights in 2001 only. Indeed the peak at $f=11.3$ in the AOV periodogram is now much more pronounced with respect to the other frequencies (Fig. 4, middle plot).

In order to test this period against the two most prominent aliases at 10.3 and $12.3 \mathrm{cyc} \mathrm{d}^{-1}$ (referring to the 2002-03 data) 
Table 4. The three principal frequencies and their statistical quantities $p_{\mathrm{c}}$ and $p_{\mathrm{d}}$ as explained in the text.

\begin{tabular}{llllllllll}
\hline \hline & \multicolumn{3}{c}{2001 all data } & \multicolumn{3}{c}{$2001-01-16+17$} & \multicolumn{3}{c}{2002 all data } \\
& $f\left[\mathrm{cyc} \mathrm{d}^{-1}\right]$ & $p_{\mathrm{c}}$ & $p_{\mathrm{d}}$ & $f\left[\mathrm{cyc} \mathrm{d}^{-1}\right]$ & $p_{\mathrm{c}}$ & $p_{\mathrm{d}}$ & $f\left[\mathrm{cyc} \mathrm{d}^{-1}\right]$ & $p_{\mathrm{c}}$ & $p_{\mathrm{d}}$ \\
\hline$f_{1}$ & $10.343(39)$ & 0.344 & 0.381 & $10.293(46)$ & 0.383 & 0.360 & $10.346(42)$ & 0.252 & 0.246 \\
$f_{2}$ & $11.340(41)$ & 0.463 & 0.432 & $11.301(40)$ & 0.532 & 0.538 & $11.362(61)$ & 0.653 & 0.597 \\
$f_{3}$ & $12.336(37)$ & 0.192 & 0.187 & $12.293(53)$ & 0.084 & 0.102 & $12.344(48)$ & 0.095 & 0.157 \\
\hline
\end{tabular}

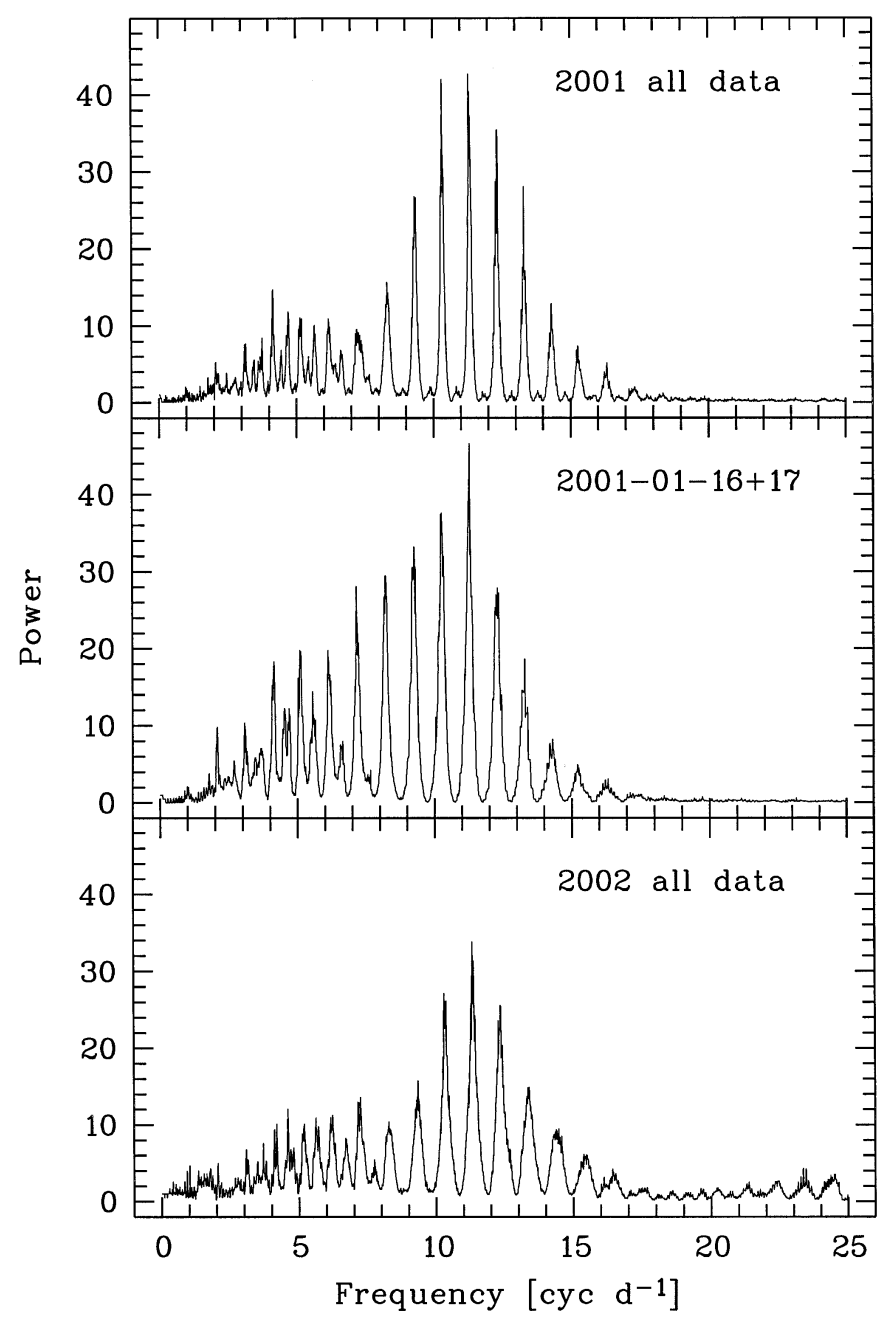

Fig. 4. AOV periodograms for the data sets as indicated in the plot. The highest peak in all periodograms corresponds to $P=2.12 \mathrm{~h}$.

we have run a Monte Carlo simulation (Mennickent \& Tappert 2001). This routine computes a thousand data sets by adding a random value to each data point, based on the variance of the sine fit to the real data. A periodogram for each of these sets is established and the frequencies and their power are recorded. Equivalently to the method outlined by Thorstensen \& Freed (1985; hereafter TF85) one can now compute a discriminatory power $p_{\mathrm{d}}$ by dividing the number of times where a specific frequency has the largest power in comparison to the other aliases by the number of total tries. The second statistical measure used by TF85, the correctness likelihood $p_{\mathrm{c}}$, can be obtained by counting the number of times when the ratio of the power of the highest peak and that of the second highest one for the

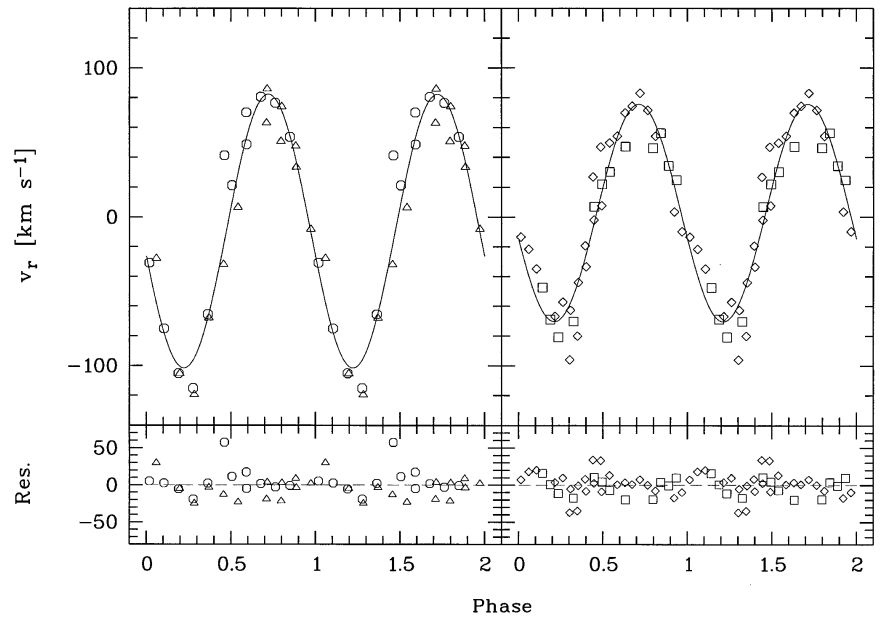

Fig. 5. Radial velocities folded on the best period $P=0.08825 \mathrm{~d}$. The left plot shows the 2001-01-16 (०) and 2001-01-17 data $(\triangle)$, the 2002-03-27 ( $\square)$ and 2002-03-28 $(\diamond)$ velocities are presented in the right plot. The respective phase zero points correspond to the values derived with the diagnostic diagram (Sect. 3.4). The best sine fit and the fit residuals are also shown.

artificial data corresponds to that of the real data within a certain range of ratios (TF85 propose a typical value of 0.1 ).

Table 4 summarises the results of this analysis for the three most prominent frequencies. The highest statistical significance for the principal frequency $f_{2} \sim 11.3 \mathrm{cyc} \mathrm{d}^{-1}$ is obtained to $p_{\mathrm{c}}=0.65$ in the 2002-03 data. This favours the identification of $f_{2}$ with the orbital frequency, but is certainly too low to provide an unambiguous decision, with $f_{1} \sim 10.3 \mathrm{cyc} \mathrm{d}^{-1}$ representing an important alias.

After the initial submission of this paper we have been informed of the research by Fenton \& Thorstensen (private communication) who obtained $P=0.0880(2) \mathrm{d}$ from time-resolved spectroscopy of GZ Cnc. They report that their run spans over $6 \mathrm{~h}$ of hour angle and does not present any daily alias problems.

This period fits very well with our best frequency $f_{2}$, which, as an average of the "quiescence" data sets, corresponds to a period

$P=0.08825(28) \mathrm{d}=2.118(07) \mathrm{h}$.

We therefore identify this period with the orbital variation.

Figure 5 presents the radial velocities folded on our period.

\subsection{Line-profile analysis}

Even for medium spectral resolution an analysis of the emission line profile is always worth undertaking, as at least it might provide a motivation for further studies. In the present case, the 


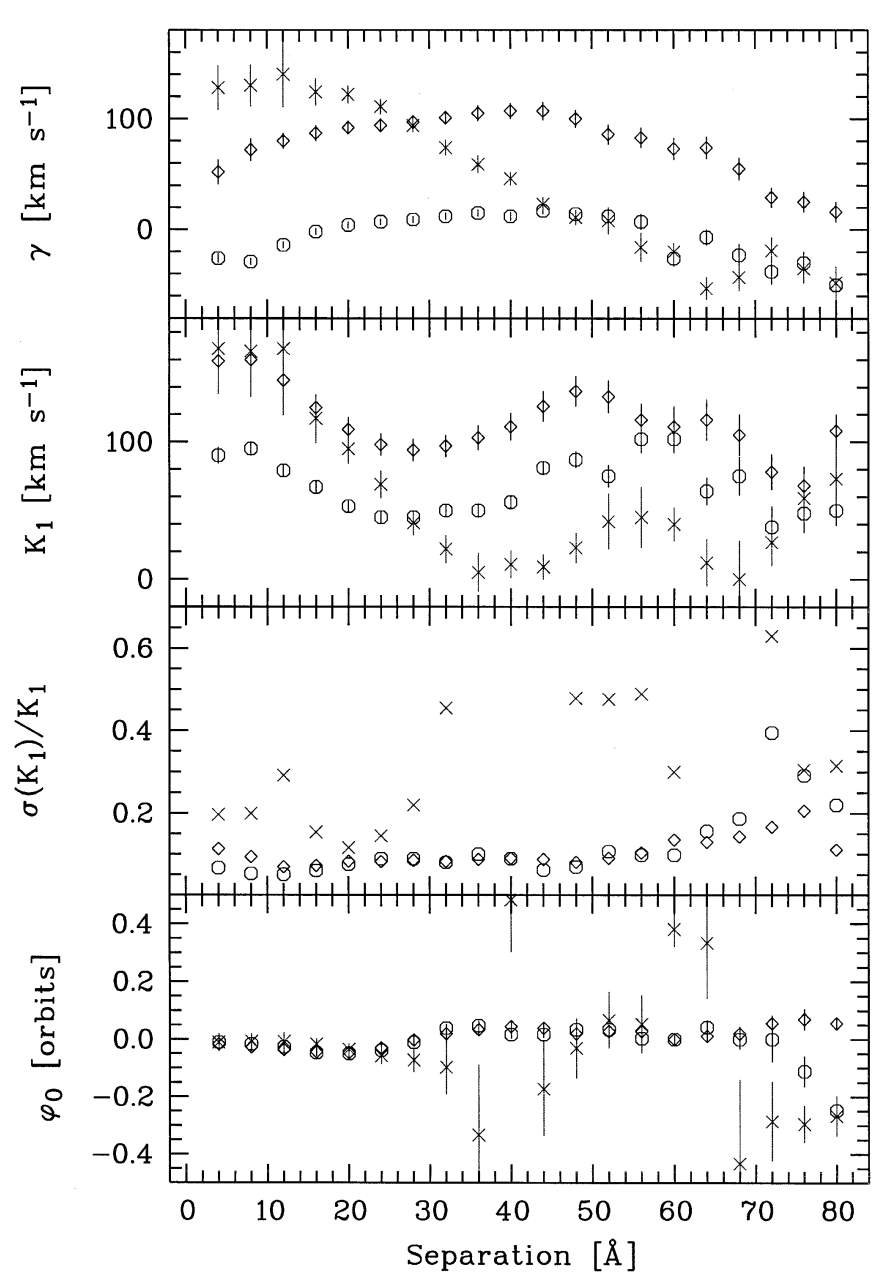

Fig. 6. Diagnostic diagrams for the following data sets: 2001-01-16+17 $(\diamond), 2001-01-18(\times)$, and 2002-03 (O). The zero point of $\varphi_{0}$ was calculated with respect to the value corresponding to a separation $d=60 \AA$ for each observing run individually (i.e., 200101-16+17 and 2001-01-18 were corrected for the 2001-01-16+17 value, the 2002-03 set for the 2002-03 value).

fact that at least one data set caught the system in a different luminosity state, and that its exclusion led to a more unambiguous determination of the orbital period, already calls for a more thorough investigation. The only really feasible line in our data for such a study is $\mathrm{H} \alpha$, as other lines are either too noisy (He I, $\mathrm{He}$ II, etc.) or are distorted by blends (basically all Balmer lines from $\mathrm{H} \beta$ on bluewards).

Due to the modest spectral and phase resolution the more advanced technique of Doppler tomography (Marsh \& Horne 1988), which in principle would yield the clearest picture on the emission distribution in the system, does not provide any significant results. However, the application of the diagnostic diagram (Shafter 1983) and the $V / R$ plot (Tappert 1999) will still give at least a rough idea of a possible additional component and of the differences between the individual data sets.

In the former method, two identical Gaussians (in the present case with $F W H M=4 \AA$ ), horizontally separated by a quantity $d$, are used to measure radial velocities of different

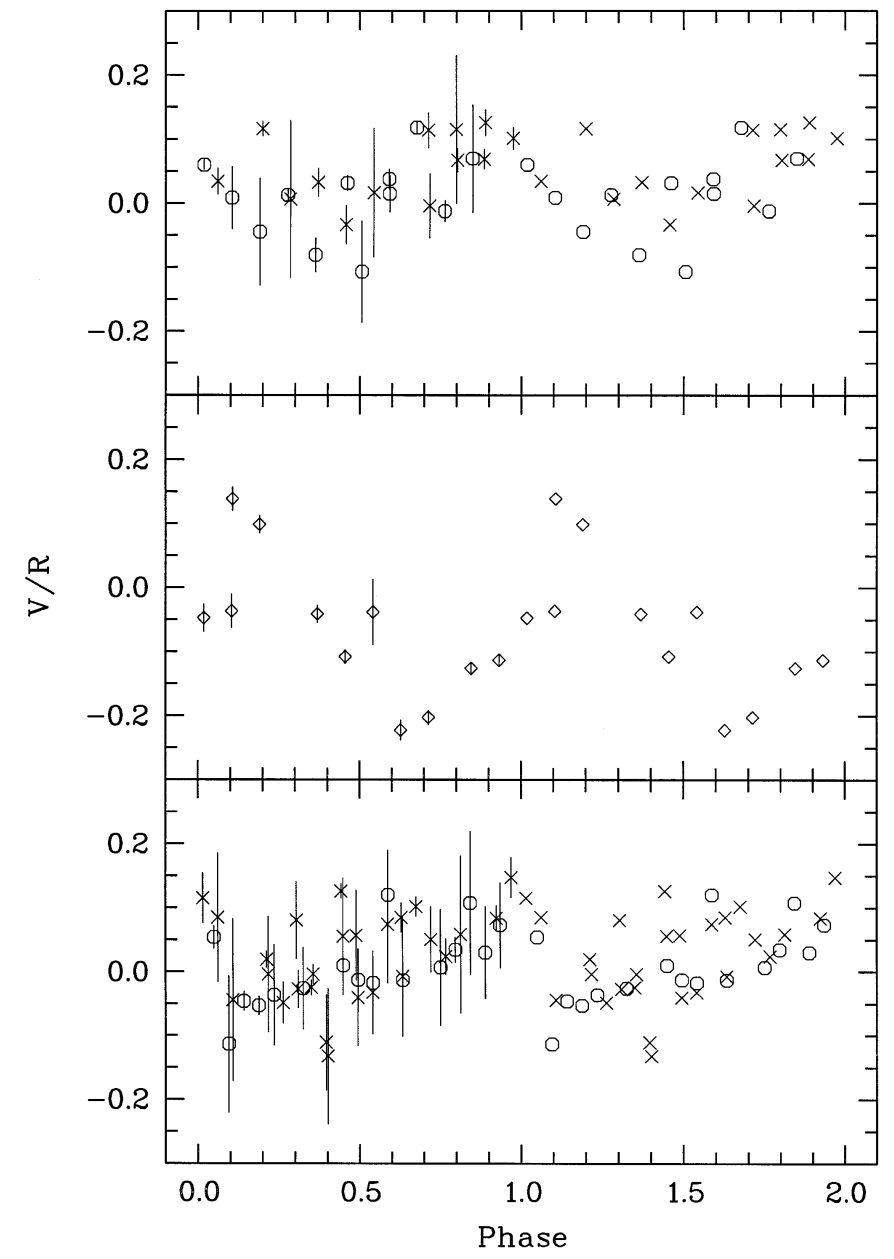

Fig. 7. $V / R$ plots for the individual data sets. Top: 2001-01-16 (O) and 2001-01-17 (×). Middle: 2001-01-18. Bottom: 2002-03-27 (O) and 2002-03-28 $(\times)$. Two phase cycles have been plotted, the first one including errors. The latter have been determined by a Monte Carlo simulation. Note that the 2001 data share a common zero phase.

parts of the emission line by variation of $d$ (Schneider \& Young 1980). The resulting velocities $v(d, \varphi)$ are fit with a function

$v(d, \varphi)=\gamma(d)-K_{1}(d) \sin (\varphi)$

The fit parameters are plotted as a function of $d$ to yield the diagnostic diagram. In order to identify the part of the line which is undisturbed from possible additional components, one usually also plots the percentage error of the semi-amplitude $K_{1}$, $\sigma\left(K_{1}\right) / K_{1}$. A sharp increase in the latter at higher separations can be interpreted as the continuum noise dominating over the signal from the emission line. The last separation before this happens, $d_{\max }$ is usually taken as that part of the line that reflects best the motion of the white dwarf, especially if the corresponding parameters tend to approach constant values. Note, that this assumes that the wings of the line profile stem from material which is symmetrically distributed within the accretion disc.

With the comparatively low resolution of our data we do not expect to be able to extract significant parameters from the diagnostic diagram, but are mainly interested in comparing the behaviour of the individual data sets. We have first computed 


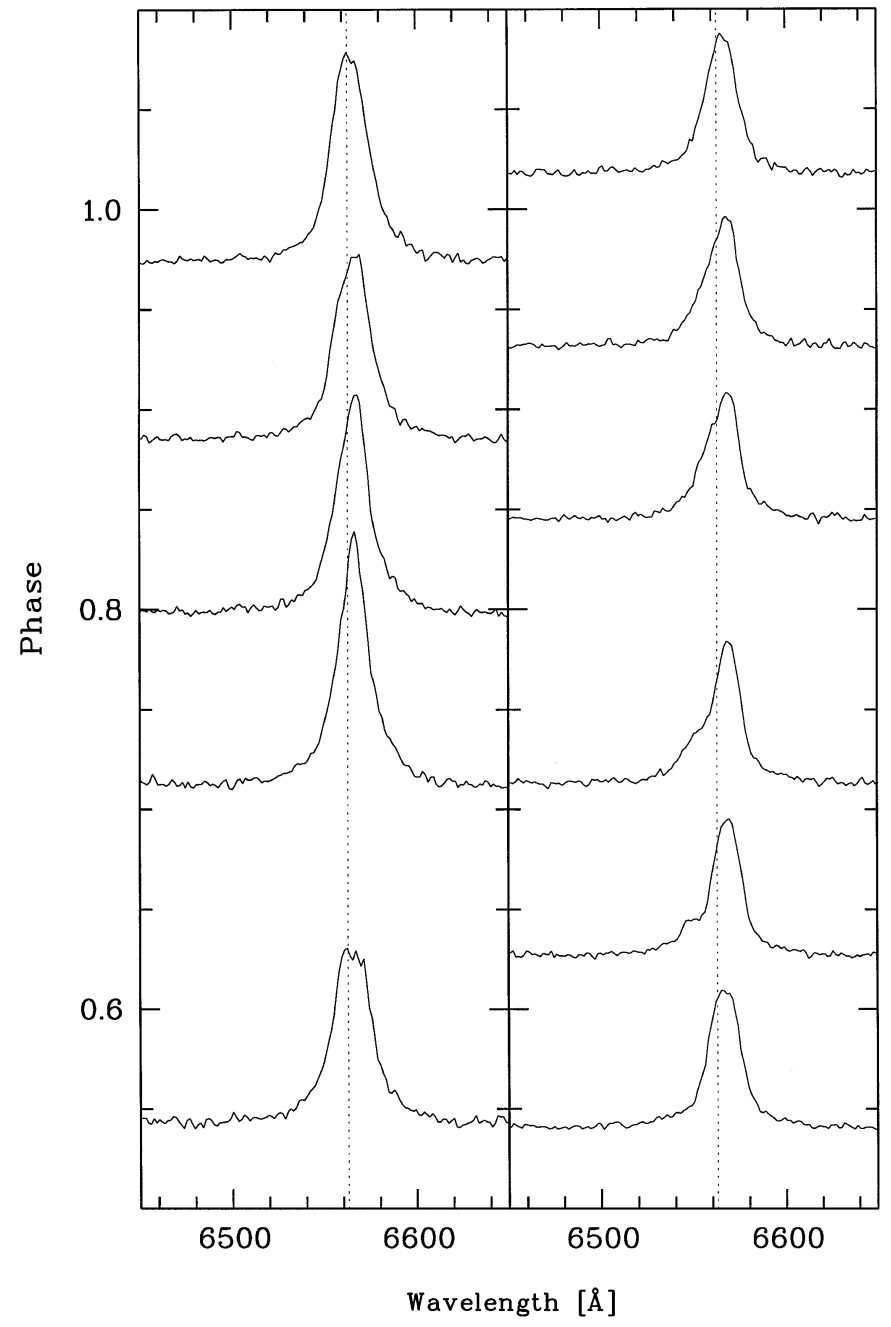

Fig. 8. Selected line profiles between phases 0.5 and 1.0 for the 2001-01-17 (left) and the 2001-01-18 data (right). The spectra have been continuum normalised, divided by 20 , and shifted vertically according to phase. The dotted line marks the rest wavelength of $\mathrm{H} \alpha$.

diagrams for each night separately. In the case of the sets from 2001-01-16 and 2001-01-17, and in the case of 2002-03-27 and 2002-03-28, the results were identical within the errors, and we therefore used the combined data sets. The resulting diagrams are shown in Fig. 6 . The $\sigma\left(K_{1}\right) / K_{1}$ curves do not really give a good indication for $d_{\max }$, and thus our choice to correct the zero point of the phase for the value corresponding to $d=60 \AA$ is somewhat arbitrary. We were originally motivated by the behaviour of the 2002-03 $K_{1}$ and $\sigma\left(K_{1}\right) / K_{1}$ curves, and for the 2001-01-16+17 data by the fact that at this point the $K_{1}$ value corresponds well to the 2002-03 $K_{1}$. However, we are not particularly interested in a specific value for $\gamma$ or $K_{1}$, and although $\varphi_{0}$ is important for the interpretation of the subsequent $V / R$ plot, it stays almost constant over the whole range of separations, and its choice therefore does not appear to be critical.

From the appearance of the curves in the diagnostic diagram we can derive two pieces of information. First, that the variation of $K_{1}$ provides evidence for the presence of an additional emission component, and second, that the similarity of the variation in all parameters of the 2001-01-16+17 and 2002-03 data sets indicates a similar type of additional emission, while there are strong differences with respect to the 2001-01-18 data.

For the $V / R$ plot the fluxes of the blue (violet) half, $F(V)$, and of the red half, $F(R)$, of the emission line profile are computed to obtain

$V / R=\log \frac{F(V)}{F(R)}$.

The outer borders of the line profile are defined by a certain intensity level $I$, the central wavelength which separates both halves is taken as the mean of the two outer wavelengths $\lambda_{V}(I)$ and $\lambda_{R}(I)$. For the present case we chose $I=0.15 I_{\max }$, with $I_{\max }$ being the peak value of the emission line.

The resulting plots, $V / R$ vs. phase, are given in Fig. 7. While the variation is very noisy and badly defined in the 2001-01-16 and 17, and the 2002-03 data, they do show a similar behaviour. In both cases a maximum at similar phases (0.9 in the upper plot, 1.0 in the lower one) can be identified. As the respective zero points of the phases were determined individually, we do not consider the observed phase difference as evidence for a fundamentally different line profile variation. The plot for the 2001-01-18 data (middle plot), however, shows a much stronger, better defined, variation, which additionally is significantly shifted in phase.

Maximum and minimum in the $V / R$ plot can usually be identified as the maximum blue- and redshift of an additional emission component. This assumes that this component is dominating the line profile and mostly confined to the centre of the profile. However, this does not seem to work for GZ Cnc. Figure 8 shows that the minimum in the 2001-01-18 data around phase 0.65 is due to a small bump in the blue wing which then moves redwards with phase. Note that the stronger part of the line profile shows very similar velocities in both the 2001-01-18 and the 2001-01-17 data.

While this additional component in $\mathrm{H} \alpha$ at first glance appears to be an emission feature, a closer investigation of all lines (Fig. 9) and a comparison with respect to unaffected phases (Fig. 10) reveals that it is actually a high velocity absorption component. It is visible in all emission lines from phase 0.54 to 0.93 , with the probable exception of He II.

As the component is most clearly seen in the He I $\lambda 5876$ line, we chose the latter to measure its velocities. Figure 11 shows that it appears at phase 0.54 at a velocity $\sim-650 \mathrm{~km} \mathrm{~s}^{-1}$, moving redwards and apparently reaching a plateau around phase 0.9 . The latter would suggest a confinement to negative velocities, but this impression is mainly caused by the first and the last data point, which certainly are the most uncertain ones (see Fig. 9). On the other hand, we find no evidence that the component ever appears at positive velocities (Fig. 12). Unfortunately, our data does not cover a second orbit, so that it remains unclear if the absorption component represents a transient or a periodic event.

\section{Discussion}

Our analysis revealed a period of 2.118(7) h, placing GZ Cnc just at the lower edge of the period gap of CVs (Fig. 13). This 


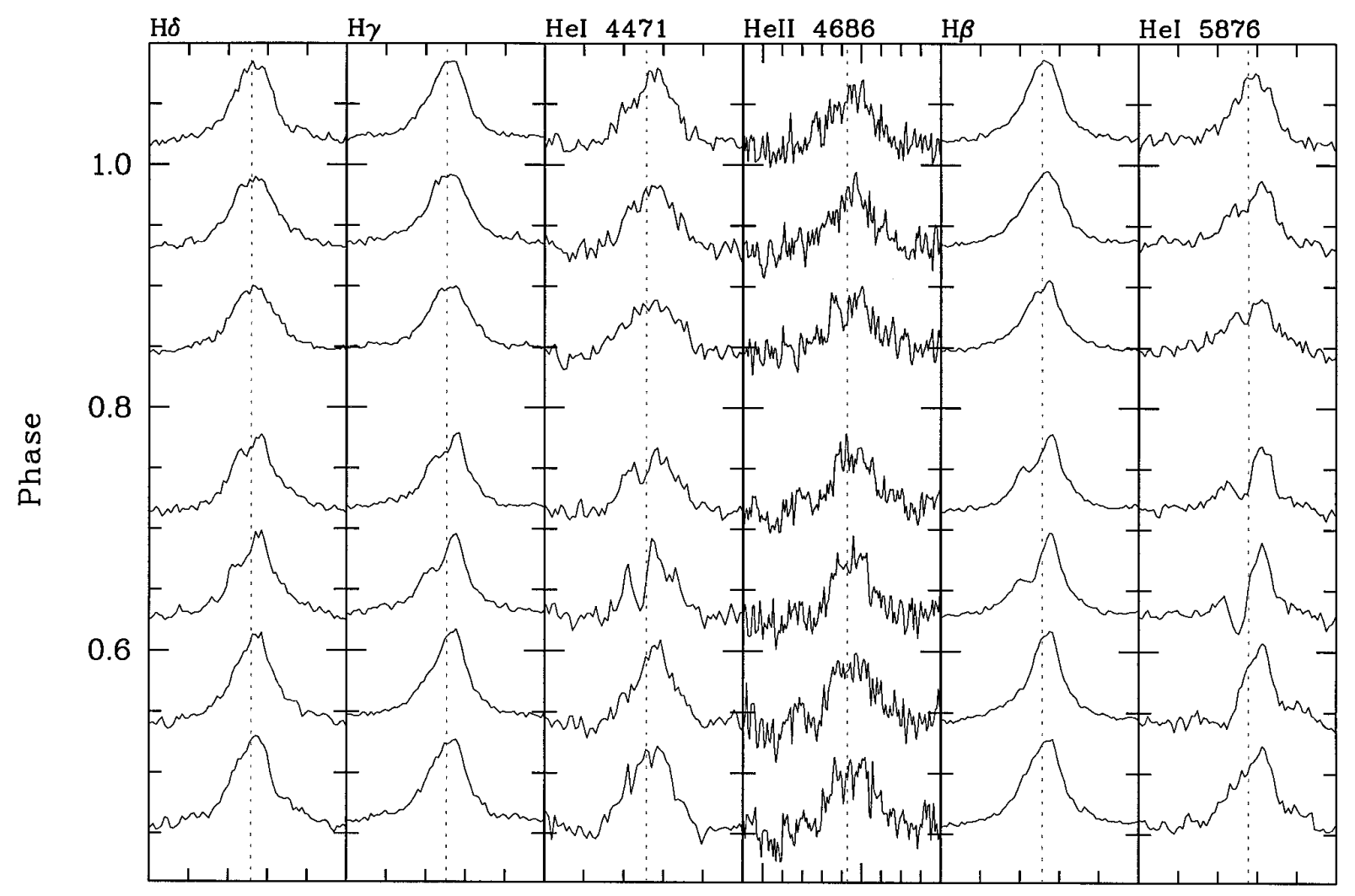

Wavelength $[\AA]$

Fig. 9. Several line profiles, as indicated in the plot, for selected phases of the 2001-01-18 data. Like in Fig. 8, the spectra have been continuum normalised, divided by a certain quantity, and shifted vertically according to phase. The dotted lines mark the respective rest wavelengths. Each unit in the wavelength axis marks $20 \AA$. Note that the wavelength range for each plot amounts to $100 \AA$, except for the much broader He II line, where $200 \AA$ are covered.

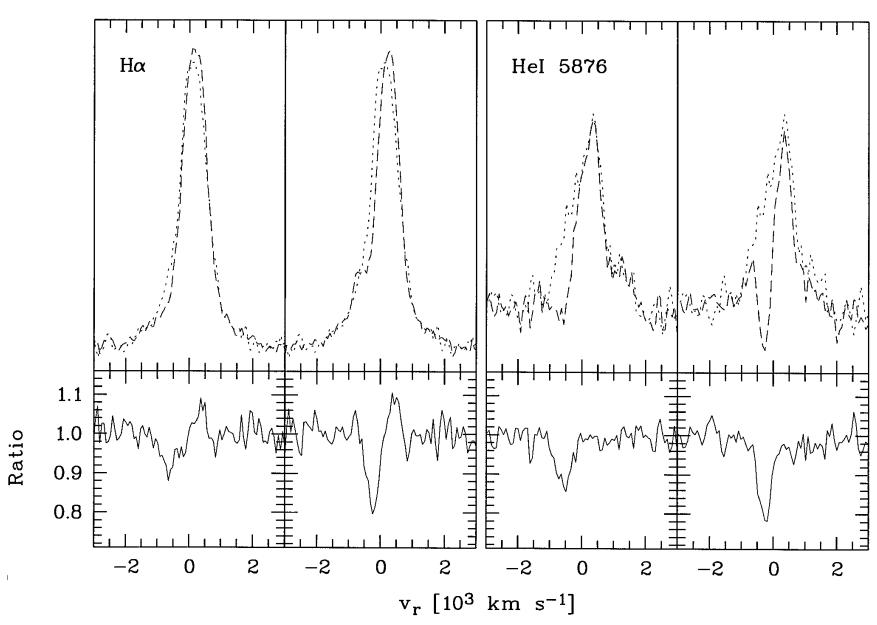

Fig. 10. $\mathrm{H} \alpha$ and He I $\lambda 5876$ line profiles of phases 0.54 (dashed, left plots of the respective line) and 0.63 (dashed, right plots) and 0.45 (dotted) on 2001-01-18. The lower plots give the ratio of the later phases with respect to phase 0.45 .

is a region which is sparsely populated, especially by dwarf novae, and it is therefore of particular interest to determine the CV subtype of the system.

Kato et al. (2002; hereafter K2002) studied the long-term behaviour, and found a similarity to those of intermediate

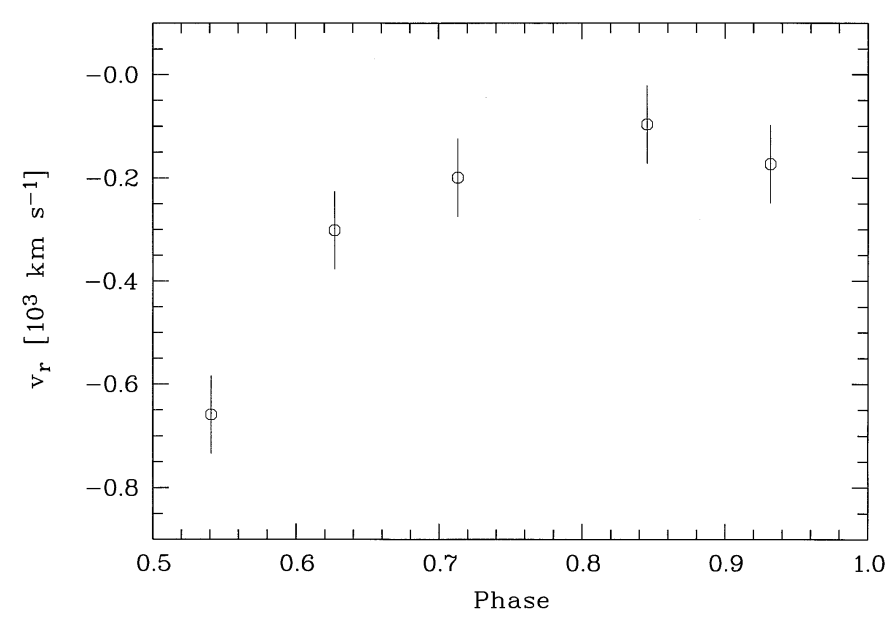

Fig. 11. Radial velocities of the He I $\lambda 5876$ absorption component as measured with a graphics cursor. The error bars refer to \pm 1 unit of the dispersion $\left(76 \mathrm{~km} \mathrm{~s}^{-1}\right)$, with the real uncertainty being probably higher at most phases.

polars (IPs) in that the outburst distribution appeared to be "clustered". Specifically the authors mention the detection of four outbursts within two months in 2002, while the only known outburst before that date has been recorded at the end of 2000. Still, our data proves that at least one brightening has 


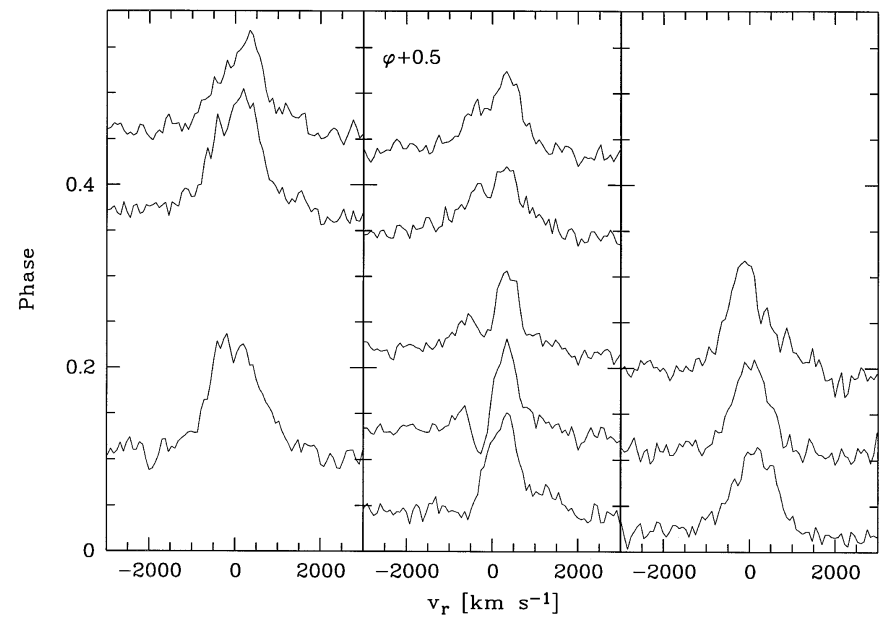

Fig. 12. All 2001-01-18 He I $\lambda 5876$ line profiles in time order from lower left to upper right. Phases between 0.5 and 1.0 are shown in the middle plot in order to facilitate comparison with opposite phases.

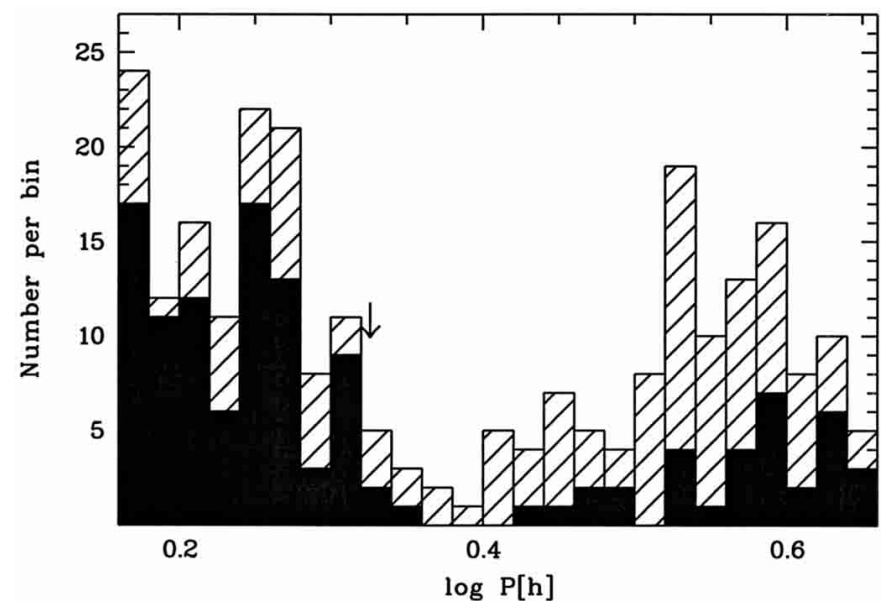

Fig. 13. The period distribution of CVs around the period gap. The hashed histogram represents the distribution of all CVs, the solid one those of dwarf novae only. The data were taken from the TPP catalogue (Kube et al. 2002) as of 2002-11-05. The arrow marks the position of GZ Cnc, which is not included in either histogram.

occurred during 2001 that remained undetected. It is therefore possible that the observed clustering is not due to a variation of the outburst frequency, but caused by a variable outburst amplitude, especially as the upper detection limit in the observations used by $\mathrm{K} 2002$ varies between $m_{\mathrm{vis}} \approx 14.8$ and 13.2, the latter being already very close to the object's maximum intensity (Fig. 1 in K2002). Note, however, that frequent outbursts of variable amplitude by no means contradict the suggested similarity to V426 Oph: it certainly fits well with the latter's long-term lightcurve (Fig. 2 in K2002).

Another point in favour of the IP scenario might be provided by the appearance of the absorption component during rise to outburst. Such features during certain phases have been observed e.g. in early outburst of the short pe$\operatorname{riod}\left(P_{\text {orb }}=1.63 \mathrm{~h}\right) \mathrm{IP}$ EX Hya (Hellier et al. 1989) and in the long period $\left(P_{\text {orb }}=4.85 \mathrm{~h}\right)$ IP FO Aqr (Hellier et al. 1990). They are furthermore a common property of the
SW Sex stars (Hellier 2000, and references therein), which have been recently proposed to represent high mass-transfer IPs by Rodríguez-Gil et al. (2001).

These features have been explained by a scenario where part of the gas stream from the secondary overflows the disc, and impacts on the inner part of the disc or connects with the magnetosphere of the white dwarf (Hellier 2000; Hellier et al. 1989). However, an important difference of the GZ Cnc absorption with respect to the above systems is that their absorption features consist of low-velocity components. A stellar wind as suggested for a similar phenomenon in the nova-like BZ Cam (Patterson et al. 1996) might represent a more probable alternative.

Another similarity with EX Hya, but also a common feature of dwarf novae, lies in the presence of an isolated emission component in the quiescence data, as suggested by the $V / R$ plot (Fig. 7). This component is confined to the line centre and could thus stem from the impact region of the gas stream with the outer accretion disc.

\section{Conclusions}

There are three pieces of evidence which might favour GZ Cnc as an intermediate polar (IP): the long-term behaviour (Kato et al. 2002), the unusual, although not exorbitant, strength of the He II line, and the appearance of the absorption component during rise to outburst. None of these is really conclusive, basically because they are based on insufficient data. However, in their sum they represent at least a strong indication.

We conclude that further observations are necessary in order to decide pro or contra an IP nature for GZ Cnc. Especially helpful would be a better sampled long-term lightcurve and high-speed photometry. If an IP nature were to be confirmed, it would make GZ Cnc a rare system indeed: the TPP catalogue (Kube et al. 2002) records only one out of twenty confirmed IPs below the period gap.

Finally, the non-uniform emission distribution in GZ Cnc marks it as an interesting system for further and more detailed studies using higher resolved spectroscopic data.

Acknowledgements. We thank Boris Gänsicke and Ronald Mennickent for advice and discussions. John Thorstensen kindly informed us on the result of Bill Fenton's and his research, which is gratefully acknowledged. We thank the referee, Pablo Rodríguez-Gil, for helpful comments and for pointing out the behaviour of highvelocity components in intermediate polars and SW Sex stars. This research has made use of the SIMBAD database, operated at CDS, Strasbourg, France.

\section{References}

Bade, N., Engels, D., Voges, W., et al. 1998, A\&AS, 127, 145

Bessell, M. S. 1990, PASP, 102, 1181

Echevarría, J. 1984, Rev. Mex. Astron. Astrofis., 9, 99

Hellier, C. 2000, New Astron. Rev., 44, 131

Hellier, C., Mason, K. O., Smale, A. P., et al. 1989, MNRAS, 238, 1107

Hellier, C., Mason, K. O., \& Cropper, M. 1990, MNRAS, 242, 250 
Jiang, X. J., Engels, D., Wei, J. Y., Tesch, F., \& Hu, J. Y. 2000, A\&A, 362,263

Kato, T., Uemura, M., Buczynski, D., \& Schmeer, P. 2001, IBVS, 5123

Kato, T., Dubovsky, P. A., Stubbings, R., et al. 2002, A\&A, 396, 929

Kube, J., Gänsicke, B. T., \& Hoffmann, B. 2002, in The physics of cataclysmic variables and related objects, ed. B. T. Gänsicke, K. Beuermann, \& K. Reinsch, ASP Conf. Ser., 261, 678

Landolt, A. U. 1992, AJ, 104, 340

Marsh, T. R., \& Horne, K. 1988, MNRAS, 235, 269

Mennickent, R. E., \& Tappert, C. 2001, A\&A, 372, 563

Patterson, J. 1984, ApJS, 54, 443

Patterson, J., Patino, R., Thorstensen, J. R., et al. 1996, AJ, 111, 2422

Ritter, H., \& Kolb, U. 1998, A\&AS, 129, 83

Rodríguez-Gil, P., Casares, J., Martínez-Pais, I. G., Hakala, P., \& Steeghs, D. 2001, ApJ, 548, L49

Scargle, J. D. 1982, ApJ, 263, 835

Schneider, D. P., \& Young, P. 1980, ApJ, 238, 946

Schwarzenberg-Czerny, A. 1989, MNRAS, 241, 153
Shafter, A. W. 1983, ApJ, 267, 222

Stetson, P. B. 1992, in Astronomical Data Analysis Software and Systems I, ed.D. M. Worrall, C. Biemesderfer, \& J. Barnes., ASP Conf. Ser., 25, 291

Takamizawa, K. 1998, VSNET Obs., No. 10504

Tappert, C. 1999, Ph.D. Thesis, Ruhr-Universität Bochum

Tappert, C., \& Hanuschik, R. W. 2001, in Astrotomography, Indirect Imaging Methods in Observational Astronomy, ed. H. M. J. Boffin, D. Steeghs, \& J. Cuypers, Lect. Notes Phys., 573, 119

Thorstensen, J. R., \& Freed, I. W. 1985, AJ, 90, 2082

Tody, D. 1993, in Astronomical Data Analysis Software and Systems II, ed. R. J. Hanisch, R. J. V. Brissenden, \& J. Barnes, ASP Conf. Ser., 52, 173

Warmels, R. H. 1992, in Astronomical Data Analysis Software and Systems I, ed. D. M. Worrall, C. Biemesderfer, \& J. Barnes., ASP Conf. Ser., 25, 115

Warner, B. 1995, Cataclysmic Variable Stars (Cambridge University Press)

Williams, G. 1983, ApJS, 53, 523 